Research Article

Mikhail Danilov* and Arkadi Karpov

\title{
A classification of meteor radio echoes based on artificial neural network
}

https://doi.org/10.1515/astro-2018-0037

Received Feb 04, 2018; accepted Sep 17, 2018

Abstract: An artificial neural network is described for classification of meteor trails into the distinct overdense, intermediate and underdense trail categories. The neural network was trained and on model data obtained using the "KAMET" program and tested on real data. The best result of classification success rate of $95 \%$ without according to the heights of the formation of meteor trails. Results of classification with according to the heights of the formation of meteor trails are $82 \%$ - 91\%.

Keywords: Meteor radio echoes, Classification algorithms, Artificial neural networks, Radiowave propagation

\section{Introduction}

In order to process a large amount of meteoric data and to clarify the parameters of radio echoes coming from meteor radars, it is necessary to be able to separate radio meteor echoes into sustainable classes that have significant differences from each other.

In the classical theory of the formation of a meteor trail, such classes of meteor radio echoes are distinguished as the classical underdense and the classical overdense. In practice, in addition to these classes, there are meteor trails of intermediate type and it is very important to allocate such meteor trails.

The observation conditions also contribute to the observations of meteor trails. It may happen that the overdense trails will look like underdense, and vice versa. This is also important to learn to determine.

To solve these problems and classification problems, it was proposed to create a system for automatic classification of meteoric echoes based on an artificial neural network, trained by the "supervised learning" algorithm. "Supervised learning" artificial neural networks have been used for classification of meteor echoes in the works (Fraser et al. 1992; Melville et al. 1996).

Corresponding Author: Mikhail Danilov: Kazan Federal University, Russian Federation, Kazan, 420008, 18th Kremloyvskaya Str., Institute of physics, Department of radio physics;

Email: mikhdan@mail.ru

Arkadi Karpov: Kazan Federal University, Russian Federation, Kazan, 420008, 18th Kremloyvskaya Str., Institute of physics, Department of radio physics; Email: arkadi.karpov@kpfu.ru
The objective of this study is to develop an automatic classifier of meteoric radio echoes based on a multi-layer artificial neural network and its application to the classification of radio echo.

\section{Methods}

The general pattern of neural network classification is well represented in the work (Karpov 1995), but to make the results more user-friendly, we will reiterate the most important general points and present the main elements of the artificial neural networks (ANN).

The ANN is the combination of neural elements and connections between them. Each ANN is based on relatively simple, mostly single-type elements (cells) imitating the work of brain neurons (Figure 1). From here on, the neuron will mean an artificial neuron, an ANN cell.

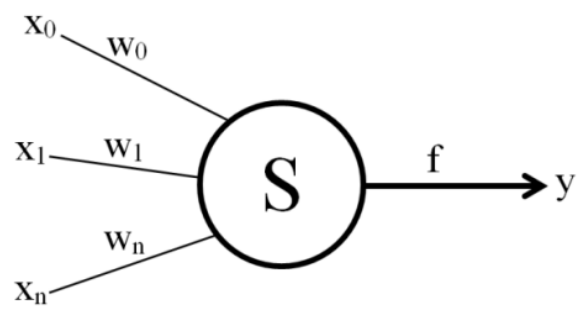

Figure 1. Neuron mathematical model.

The neuron has a group of synapses - unidirectional input connections connected to the outputs of other neurons. Each synapse is characterized by the value of synap-

¿ Open Access. (C) 2018 M. V. Danilov and A. V. Karpov, published by De Gruyter. 
tic communication or its weight $\mathrm{w}_{i}$. The neuron has a current state which is usually defined as the weighted sum of its inputs:

$$
s=\sum_{i=1}^{n} x_{i} w_{i}
$$

The neuron has an axon - the output connection of this neuron from which the signal goes to the synapses of the next neurons. The neuron's output is its state function. $y$ $=f(\mathrm{~s})$. The function $f(\mathrm{~s})$ is called activation function. Typically, three main activation functions are used:

sigmoid function:

$$
f(x)=\frac{1}{1+\exp (-\alpha x)}
$$

bipolar sigmoid function:

$$
f(x)=\frac{2}{1+\exp (-\alpha x)}-1
$$

threshold function

$$
f(x)=\left\{\begin{array}{l}
1, x \geq 0, \\
0, x<0
\end{array}\right.
$$

Many of the ANN neurons can be divided into subsets - layers. Neurons interact layer through layers. The artificial neural network layer represents a set of neurons that receive signals from other neurons within this network within a time step simultaneously. A simple neural network diagram with one hidden layer is shown in Figure 2.

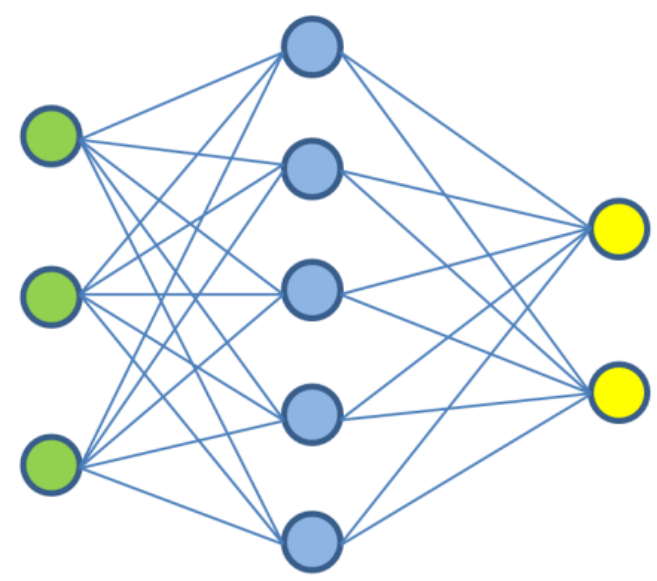

Figure 2. A simple neural network diagram with one hidden layer.

The ANN training process lies in adjusting the weighing ratios of the neurons when applying training sample patterns to the ANN input. The training is iterative when all examples from the training set are presented in one iteration during the training process. The training sample represents a set of vectors of indicators and their corresponding resulting vectors coding the meteor radio reflection class. Each pattern vector of the indicators from the training sample is set to the input of the network, processed inside the ANN structure resulting in an output signal that is compared to the corresponding resulting vector value from the training sample. Then, a specific rule calculates the error, the weighing ratios of the connections within the network are altered depending on the selected training algorithm. The learning set vectors are applied to the ANN input sequentially, errors and metrics (for example, accuracy - the percentage of correct algorithm responses) are calculated for training and test samples, and weights are adjusted for each vector until the error in the whole learning array reaches an acceptable low level.

The number of inputs of the neural network equals the number of indicators for the classification. The number of outputs equals the number of meteor radio reflection classes for the given classification. The double-layer neural networks were implemented using the Anaconda 4.4.0 distribution for Python 3.6 programming language and the Deep Learning library Keras 2.0.2. Table 1 shows the main ANN parameters used in the work.

Table 1. The parameters of the artificial neural networks used in the process.

\begin{tabular}{ccccc}
\hline $\begin{array}{c}\text { ANN } \\
\text { designation in } \\
\text { the present } \\
\text { article }\end{array}$ & $\begin{array}{c}\text { Number } \\
\text { of } \\
\text { network } \\
\text { inputs }\end{array}$ & $\begin{array}{c}\text { Layer } \\
\text { number }\end{array}$ & $\begin{array}{c}\text { Number } \\
\text { of } \\
\text { neurons }\end{array}$ & $\begin{array}{c}\text { Number } \\
\text { of } \\
\text { network } \\
\text { outputs }\end{array}$ \\
\hline A & $3(4)$ & 1 & 300 & 2 \\
B & 6 & 2 & 18 & \\
C & 6 & 1 & 120 & 3 \\
D & 6 & 1 & 102 & \\
E & 6 & 1 & 80 & 3 \\
F & 6 & 2 & 80 & 3 \\
& 6 & 1 & 100 & 3 \\
& & 2 & 37 & 3 \\
\hline
\end{tabular}

The number of neurons in the hidden layers was set by "rule 2-5", which states that the number of connections between the network's neurons should be $2-5$ times less than the number of examples in the training sample and based on the equation employed for determining the number of 

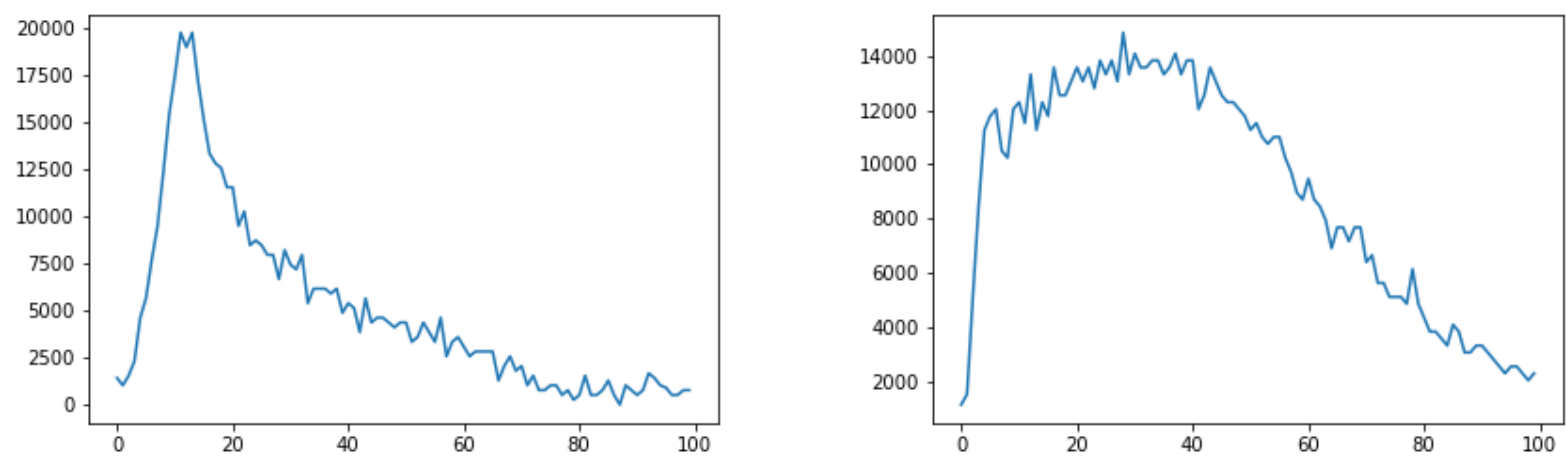

Figure 3. Examples of meteor echoes ATC: Underdense and overdense classes.

weighting ratios

$$
N_{W}=(M+1) m_{1}+\sum_{j=2}^{L}\left(m_{j-1}+1\right) m_{j}+\left(m_{L}+1\right) K
$$

where $N_{W}$ - represents the number of weighting ratios (number of connections between neurons), $M$ is the number of inputs, $K$ is the number of outputs, $L$ is the number of layers of the neural network, $\mathrm{m}_{i}$ is the number of neurons in layer $\mathrm{i}$.

Of the training sample, $25 \%$ of the data was allocated for testing.

The works (Fraser et al. 1992; Melville et al. 1996) use three reflection classes: echoes from underdense trails, echoes from overdense trails, echoes from trails and echoes of non-meteor origin. Underdense trails are trails with a linear electron concentration on the axis of the trail equal to $\alpha<2,4 \cdot 10^{14}$ electron $/ \mathrm{m}$. The falling radio-wave is reflected without the effects of the secondary electrons re-radiation and and radio reflection ATC from such a classical underdense trail following a rapid growth of the amplitude has an exponential drop due to the recombination of free electrons and trail ions. An overdense trail is a trail with a linear electronic concentration of $\alpha>2,4 \cdot 10^{14}$ electron $/ \mathrm{m}$. The falling wave is reflected from the trail as through from the metal cylinder of some critical radius. The form of the reflection ATC from the classic overdense trail is as follows: After a rapid growth, the reflection amplitude slowly increases (because of the growth of the critical radius due to ambipolar diffusion) and slows down to zero after having reached its maximum. Examples of such echoes are presented in Figure 3.

This two-stage classification system gives rise to certain problems as many meteor trails can in practice be attributed to both underdense and overdense.
In this work, we are also looking at two classes of echoes: from underdense trails and overdense trails. Intermediate-class trails are also added which may be classified as underdense trails and overdense trails. The number of classes is expected to go up later on but the introduction of new classes should not affect the training sample formation process.

This work has made use of the indicators characterizing the ATC profile that we proposed in the work (Kartashova et al. 2017). A "set of indicators 2 " has also been formed consisting of the ATC profile width at 10\%, 50\% and $90 \%$ of the maximum amplitude value. This data is graphically shown in Figure 4.

The ATC profile widths for the classification have been selected due to the following considerations: Radio reflection ATC from such a classic udnerdense trail following a rapid growth of the amplitude has an exponential drop due to the recombination of free electrons and trail ions. It is typical for an overdense trail that after a rapid growth, the reflection amplitude slowly increases (due to the growth of the critical radius due to ambipolar diffusion) and slows down to zero after having reached its maximum. It is clear that the ATC profile widths for different types at different levels from the amplitude will vary.

Selecting data to train the network and processing it is the most complex classification phase. The training dataset must meet several criteria:

- Representativeness - the data must illustrate the true state of affairs in the subject domain;

- Consistency - conflicting data in the training sample will result in poor network training quality.

The traditional approach to preparing the training sample is the first to be considered. In the works (Fraser et al.1992; Melville et al.1996), a set of training samples is put together by selecting patterns from the array of experimen- 


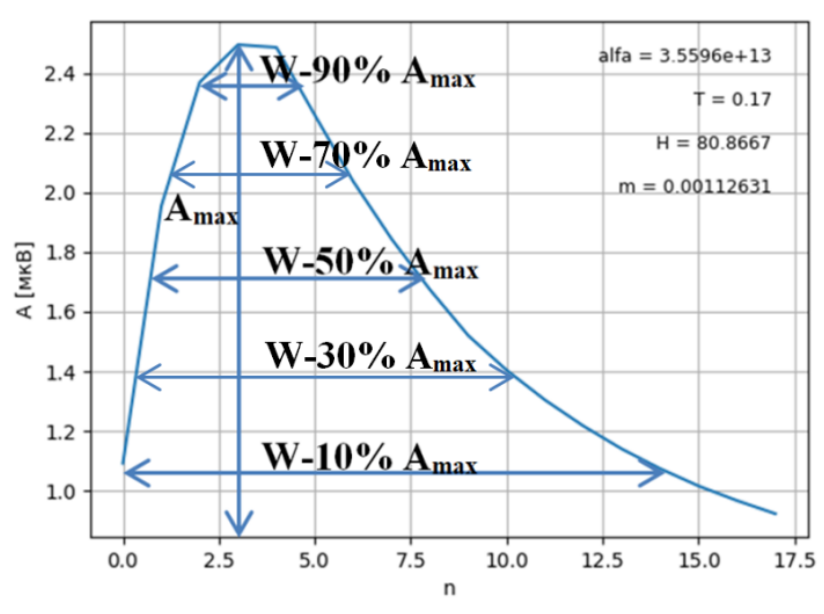

Figure 4. Indicators for classification.

tal data. This approach has a number of drawbacks that may lead both to errors in the training of neural networks and to difficulties in using a sample without significantly processing the data when the number of classes changes.

The main metric of ANN performance is accuracy - the ratio of the number of correctly classified ATC to the number of all ATC in the test sample. In general, the test sample adds up to 25 per cent of patterns selected randomly from the training sample. In addition, during the ANN training, 2 metrics are automatically calculated in the classifier based upon the Keras library: the training sample accuracy (train) and the testing sample accuracy (test) depending on the training iteration. This dependence shows the extent of accuracy which was achieved during the ANN training process. It also allows for checking whether there has been a retraining where the ANN only "remembered" the patterns from the training sample producing incorrect results in operation mode. In this case, the testing sample accuracy will be higher than the training sample accuracy.

Let us consider the issues that arise when implementing a traditional approach in creating a training sample.

Firstly, in order to obtain acceptable accuracy in the classification of meteor radio echoes by artificial neural networks, there is a need for samples of training patterns that represent as many as possible examples of meteor radio echoes for pre-defined classes. This is possible in the case of large volumes of such samples. As a result, if there is a need to clarify the classification and to increase the number of classes, it is necessary to prepare the training sample again which becomes extremely expensive. In practice, it is not always possible to obtain large amounts of training samples or all possible examples to train neural networks resulting in a non-representative sample. Therefore, the accuracy of the classification is directly related to

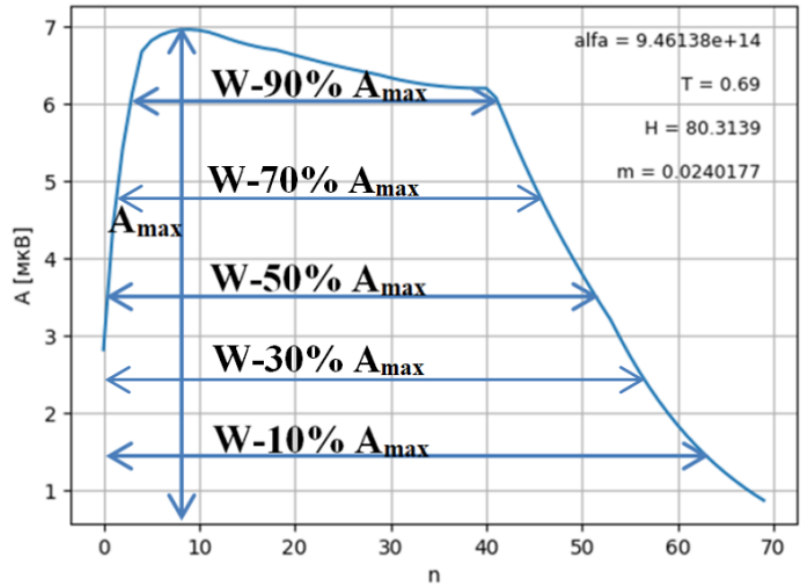

the size of the training sample and the relatively quick creation of the training sample is a very time-consuming task. Also, when introducing additional classes, one must fully re-design the existing training sample.

Another problem in preparing training samples is the introduction of subjective errors in the classification by decisions based on experts' opinions. Traditionally, in classifying meteor radio-echoes, the ATC which belong to the general experimental collection are used as the training sample and, in the opinion of the authors (experts), characterize a particular type of echoes to the best advantage. In this case, the training sample will be based on echoes which, due to the influence of noise or observing conditions, may be attributed to another class by an expert and the percentage of such errors, in light of the abovementioned reasons, may be quite high. This results in conflicting data in the training samples. However, even the high accuracy of training only points to the fact of the training itself and not to the accuracy of the classification.

Therefore, samples of meteor radio reflection patterns that do not contain such problems are needed. These samples can be obtained by using the meteor radio-channel model through forming a training sample by modeling the ATC of meteor radio echoes. While modeling, one may set a classification and generate samples as voluminous as desired and avoid subjective errors in the definition of radio echoes classes.

This work has implemented the approach to the formation of the training sample based on the use of the modeling results of the KAMET advanced meteor radiochannel simulation model (Karpov 1995; Sulimov et al. 2017) based on the strict numerical solution of the task of radio-waves diffraction on the meteor trail. The KAMET model simulates a complete list of processes, ranging from 
the intrusion of a meteor particle into the atmosphere to the registration of the radio reflection from the trail that it has generated. KAMET generates meteor particles of random masses and velocity vectors, and then it consistently simulates the formation and ionization processes of the meteor trail, radio-wave scattering on it and the processes of its gradual degradation. As compared to other meteor channel models developed abroad (Desourdis et al. 1993), the KAMET has two substantial advantages.

Firstly, the modeling is performed through tables of the inflow of sporadic meteors into the Earth's atmosphere based on observations by the Kazan meteor radar. Secondly, the calculation of ATC is based on a strict solution (by numerical methods) of the task of electromagnetic waves diffraction on the plasma of the meteor trail (Sulimov et al. 2017). This allows for a more accurate classification of the radio echoes as the "classic" approach yields good results for underdense trails a with linear electronic concentration on the axis of the trail much smaller than $2.4 \cdot 10^{14}$ electron $/ \mathrm{m}$ and for overdense trails with a linear electronic concentration of considerably more than $2.4 \cdot 10^{14}$ electron $/ \mathrm{m}$. The intermediate area, echoes from trails with a linear electronic concentration in the range of $\left\{0,5 \cdot 10^{14}-5 \cdot 10^{14}\right\}$ can not in principle be described in the classical approach.

\section{Results}

In the learning process, the vectors of the previously selected indicators are fed to the neural network input. This data changes the weights of the hidden layers of the network in accordance with the output vector. Thus, input vectors are associated with the output vector through a change in the weighting coefficients of the neurons. The network is tested when a sample that is not involved in training is submitted to the input and the result of the ANN operation is compared with the previously known output vector.

For the beginning, a variant of the classification of meteor radio echoes without heights separation was chosen. For training, a sample of ATC meteor radio echoes obtained by modeling on a computer model of "KAMET" (Karpov 1995). To classify such a sample without dividing by heights, the neural network A was selected (Table 1). The ANN training was performed on a sample of 27.000 simulated meteor radio echoes ATC. The quality test was conducted on a sample of 9.000 modeled ATC of meteor radio echoes outside the training sample. Maximum amplitude and the ATC profile widths were used as indica- tors for the neural network A for the two classes at levels of $10 \%, 50 \%$ and $90 \%$ of the maximum amplitude value (Figure 4). Upon the conclusion of the training and quality test of the model sample, the classification accuracy result obtained was $95 \%$. The training accuracy's dependence on the training iteration is shown in Figure 5.

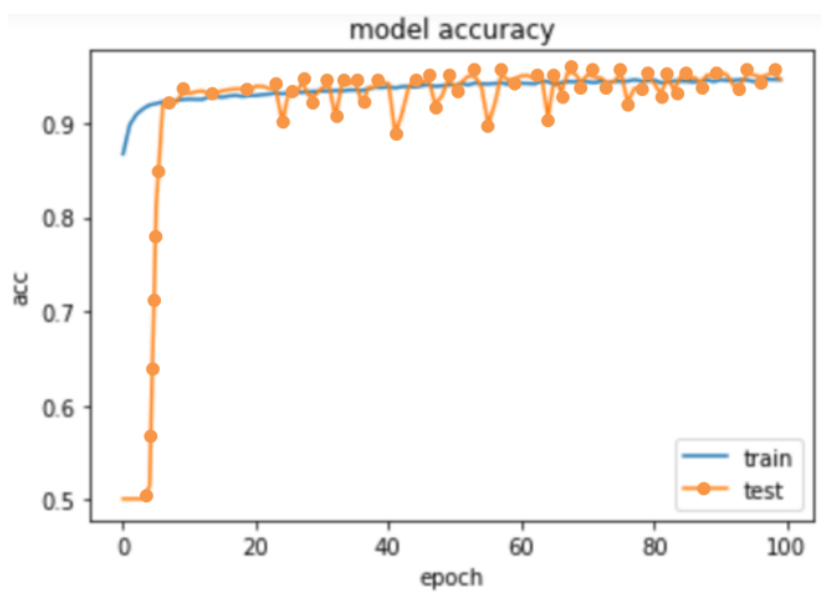

Figure 5. The training accuracy quality of the double-layer ANN for the two classes for the set of indicators from the ATC profile widths at levels of $10 \%, 50 \%$, and $90 \%$ of the maximum amplitude value and maximum amplitude.

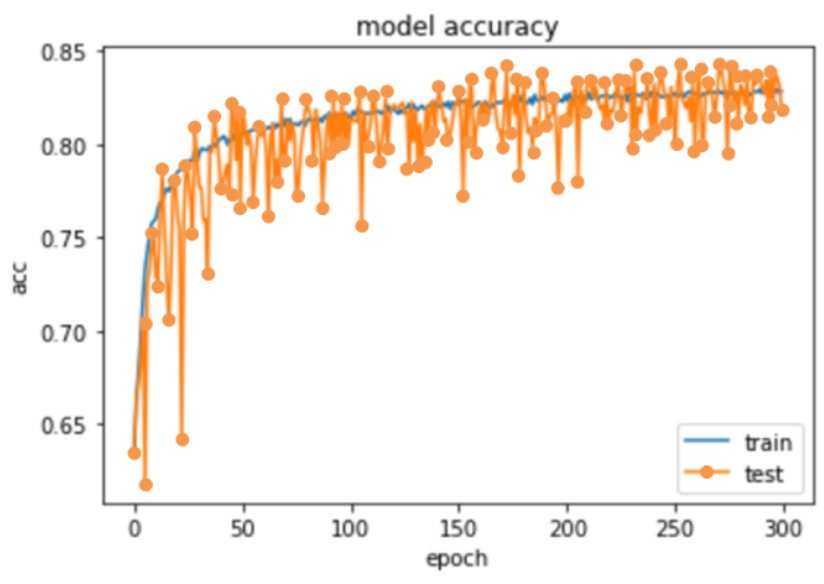

Figure 6. The training accuracy quality of the double-layer ANN for the two classes for the set of indicators from the ATC profile widths at levels of $10 \%, 30 \%, 50 \%, 70 \%$ and $90 \%$ of the maximum amplitude value and maximum amplitude.

Further, the number of indicators was increased for classification into three classes: underdense, intermediate and overdense. To classify such a sample without dividing by heights, the neural network B was selected (Table 1). The ANN training was performed on a sample of 40.500 

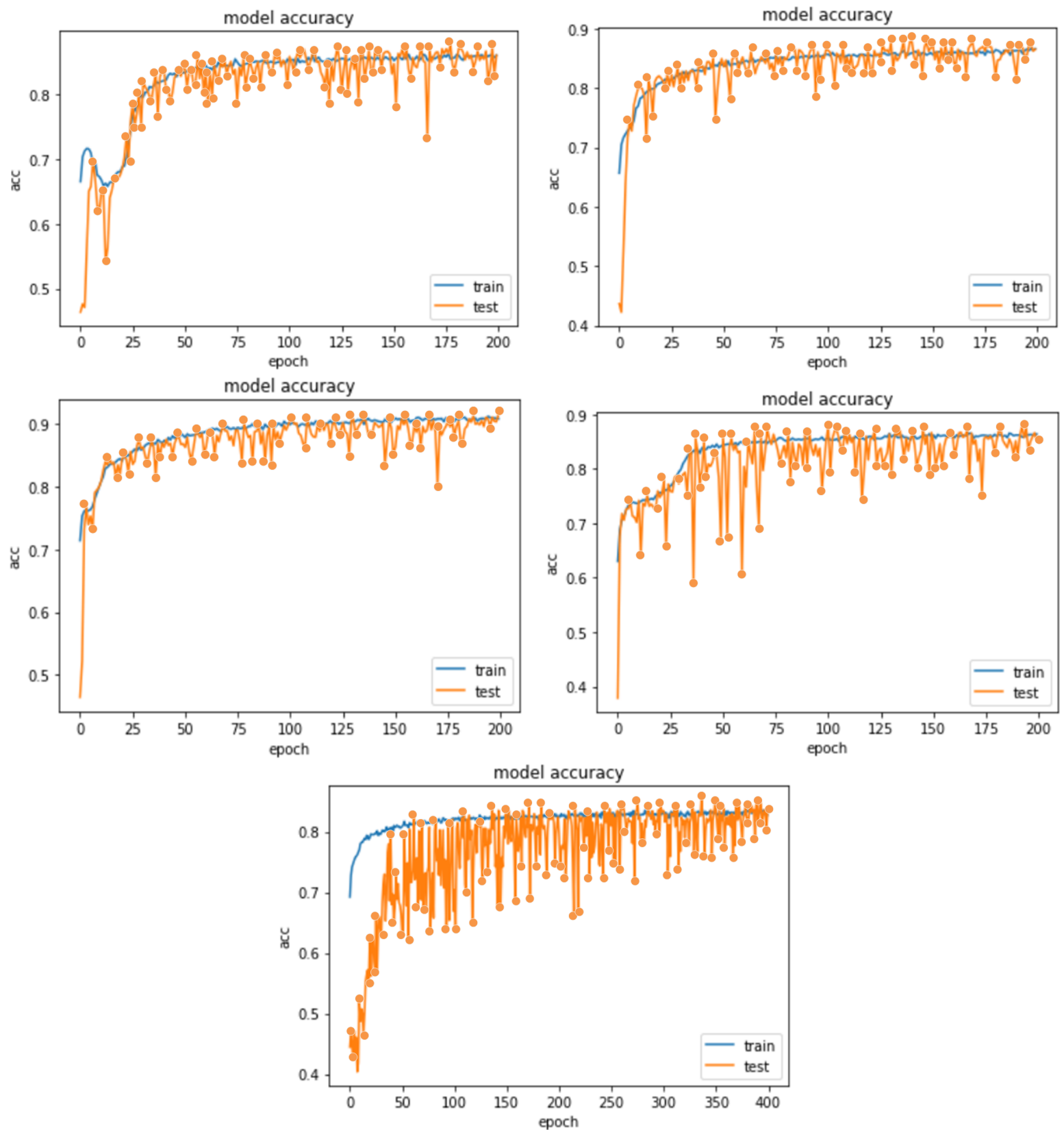

Figure 7. The training accuracy qualities of the double-layer ANN for the two classes for the set of indicators from the ATC profile widths at levels of $10 \%, 30 \%, 50 \%, 70 \%$ and $90 \%$ of the maximum amplitude value and maximum amplitude with heights separation.

simulated meteor radio echoes ATC. The quality test was conducted on a sample of 13.500 modeled ATC of meteor radio echoes outside the training sample. Maximum amplitude and the ATC profile widths were used as indicators for the neural network A for the two classes at levels of $10 \%, 30 \%, 50 \%, 70 \%$ and $90 \%$ of the maximum amplitude value (Figure 4). The classification accuracy result obtained was $82 \%$. The training accuracy's dependence on the training iteration is shown in Figure 6.
The next step in the classification process was the height separation of the sample of simulated and experimental ATC. For this step, the model data for different altitude ranges from 75 to $100 \mathrm{~km}$ in steps of $5 \mathrm{~km}$ were trained and tested, and then experimental ATC were classified. Maximum amplitude and the ATC profile widths were used as indicators for the neural network A for the two classes at levels of $10 \%, 30 \%, 50 \%, 70 \%$ and $90 \%$ of the maximum amplitude value (Figure 4). The main results are shown in Table 2. 
Table 2. The results of the classification of ATC with heights separation.

\begin{tabular}{cccc}
\hline $\begin{array}{c}\text { Range of } \\
\text { heights, } \\
\text { km }\end{array}$ & $\begin{array}{c}\text { Used ANN } \\
\text { (Table 1) }\end{array}$ & $\begin{array}{c}\text { Number of ATC in } \\
\text { the training sample }\end{array}$ & $\begin{array}{c}\text { Accuracy of } \\
\text { training and } \\
\text { testing }\end{array}$ \\
\hline $75-80$ & C & 2619 & $86 \%$ \\
$80-85$ & C & 12858 & $91 \%$ \\
$85-90$ & D & 13317 & $91 \%$ \\
$90-95$ & E & 18279 & $86 \%$ \\
$95-100$ & F & 16476 & $82 \%$ \\
\hline
\end{tabular}

The training accuracy's dependences on the training iteration with heights separation is shown in Figure 7.

Figures 5-7 show that the accuracy metrics for the training and testing samples are comparable and that no ANN retraining is observed.

32 classification indicators have been used in the work (Sulimov et al. 2017). That said, the highest accuracy of 97 percent was achieved for a single-layer ANN trained on an experimental ATC sample by the back propagation method. This work has used 4 indicators and a model sample training allowing for the use of as many ATCs as possible. The comparable classification accuracy of $95 \%$ was obtained. Thus, a smaller number of indicators makes the classification accuracy comparable to that of the work (Sulimov et al. 2017).

The preliminary results of the classification of meteor radio echoes obtained in 1994 are given in Table 3.

Table 3. The number of meteor radio reflections of various classes obtained using a classifier based on an artificial neural network.

\begin{tabular}{cc}
\hline Class of meteor radio echo & Number of meteor radio echoes \\
\hline Underdence & 6807 \\
Intermediate & 35118 \\
Overdence & 17253 \\
\hline
\end{tabular}

\section{Discussion}

Two approaches to the formation of a training sample for artificial neural networks for the classification of meteor radio-echoes have been reviewed in this work. Also the drawbacks of the traditional approach which may lead to errors in the training and in the ANN classification have been considered. The approach to the preparation of a training sample based on the use of modeling results given by the meteor radio-channel computer model "KAMET" is free of these drawbacks and allows for a quick change of the classification and for obtaining a sample of any size. This approach has faced next to no challenge in training the ANN for two and three classes with different numbers of indicators. The results of the ANN training quality test were comparable to the results of the classification accuracy in the works (Sulimov et al. 2017; Haykin 2009) where the training sample was generated from experimental data. For instance, in order to classify from two classes a set of indicators from the profile widths at different levels from the maximum amplitude and maximum amplitude yield the best result of $95 \%$ comparable to the result achieved in the work (Sulimov et al. 2017) with a larger number of indicators. In order to classify from three classes with the same set of indicators, the training accuracy quality became somewhat worse - $82 \%$. The separation of the training sample into groups according to the heights of the formation of meteor trails makes it possible to obtain the accuracy of testing the quality of instruction from $82 \%$ to $91 \%$. The best accuracy is obtained for a range of heights of 80 to $90 \mathrm{~km}$. These results are also comparable with the results obtained in (Melville et al. 1996) on a larger number of indicators.

Also it is proposed to add new classes in this classification system later on. In astronomical applications, it is of interest to accentuate the disintegrating meteoroid class. A proper accentuation of fading echoes would increase the capacity of the meteor-burst communication systems.

Another reflection class is the so-called nonreciprocal meteor echoes. The meteor synchronization and cryptography systems measure the signal phase at both points of the communication system $\varphi 1 \varphi 2$. A measurement error is maximum for so-called non-reciprocal “meteor echoes" (Sulimov 2017). It is also very important to be able to identify and exclude phase non-reciprocal echoes online by measuring the amplitude.

Acknowledgment: This work was funded by the Russian Federation government program of Competitive growth of Kazan Federal University and by the subsidy of Kazan Federal University according to the government assignment in the sphere of scientific activities (No. 3.7400.2017/8.9).

\section{References}

Fraser, D.D., Khan, Z., Levy D.C., 1992, Proceedings ICANN-92 (September 1992, Brighton, United Kingdom), 1155-1158. Melville, S., Sutcliffe, G., Fraser, D.D., 1996, Proceedings of the $4^{\text {th }}$ Pacific Rim International Conference on Artificial Intelligence (26-30 August 1996, Cairns, Australia), 423-434. 
Karpov, A.V. 1995, Radiophysics, 38, 767-773.

Sulimov, A.I., Karpov, A.V., Lapshina, I.R., Khuzyashev, R.G., 2017, IEEE Transactions on Antennas and Propagation, 65, 20092019.

Haykin, S., 2009, Neural Networks and Learning Machines, $3^{\text {rd }}$ ed., New Jersey, USA: Pearson Education.
Kartashova, A.P., Danilov, M.V., Karpov, A.V., 2017, Proceedings ACM 2017 (10-14 April 2017, Montevideo, Uruguay), 421.

Desourdis, R.J., Sidorov, V.V., Karpov, A.V., Huziashev, R.G., Epiktetov, L.A., Brown D.W., 1993, IEEE MILCOM 1993 Conference Proceedings, 32-36.

Sulimov, A.I., 2017, Proceedings RSEMW-2017, (26-30 June 2017, Gelendzhik, Russia), 1-4. 